\title{
tic\&société
}

Vol. 10, $N^{\circ} 1 \mid 1$ er semestre 2016

Contrôle social, surveillance et dispositifs numériques

\section{La déconnexion temporaire à Facebook : entre le FOMO et l'intériorisation douce du contrôle social}

\author{
Mary Jane KWOK CHOON
}

\section{(2) OpenEdition \\ 1 Journals}

Édition électronique

URL : http://journals.openedition.org/ticetsociete/2019

DOI : 10.4000/ticetsociete.2019

\section{Éditeur}

Association ARTIC

\section{Référence électronique}

Mary Jane KWOK CHOON, «La déconnexion temporaire à Facebook : entre le FOMO et l'intériorisation douce du contrôle social», tic\&société [En ligne], Vol. 10, № 1 | 1er semestre 2016, mis en ligne le 15 octobre 2016, consulté le 01 mai 2019. URL : http://journals.openedition.org/ticetsociete/2019 ; DOI : 10.4000/ticetsociete.2019

Ce document a été généré automatiquement le 1 mai 2019. 


\title{
La déconnexion temporaire à Facebook : entre le FOMO et l'intériorisation douce du contrôle social
}

\author{
Mary Jane KWOK CHOON
}

Nous remercions notre directeur de thèse, Éric George, et les deux évaluateurs anonymes pour leurs retours sur ce texte. Toute notre reconnaissance va à notre collègue Luises Vera qui a traduit le résumé en espagnol.

\section{Introduction}

1 Les médias sociaux sont une famille d'applications qui offrent aux usagers la possibilité de participer à la production et au partage du contenu en proposant différentes interfaces qui sont plus ou moins faciles d'utilisation. Parmi ce groupe d'applications, nous pouvons inclure les wikis, des sites de jeux virtuels, des mondes sociaux virtuels, des réseaux socionumériques (RSN), des blogs (Kaplan et Henlein, 2010). Les RSN sont aussi des espaces où se pratiquent différentes formes de surveillance ${ }^{1}$, notamment celle dite institutionnelle et sociale (Kwok Choon et Caron, 2012). Ainsi, les propriétaires de ces sites récoltent les données personnelles des usagers pour des raisons mercantiles. Par ailleurs, avec les révélations de Snowden, nous avons eu la confirmation que la National Security Agency (NSA, Agence nationale de la sécurité) scrute les profils des citoyens (Bennett et al., 2014). Nous notons également que les usagers épient les activités de leurs amis sur ces sites (Marwick, 2012). Ces pratiques peuvent mettre en danger la vie privée. Par exemple, le site Facebook a fait l'objet de plusieurs controverses en la matière au fil des années. Il fut accusé entre autres de tracer les activités des usagers à l'intérieur et à l'extérieur du site grâce au logiciel Beacon et de transmettre aux propriétaires de certaines applications des informations permettant d'identifier personnellement les utilisateurs (Chander, 2012). Par moments, ces pratiques peuvent nuire à la réputation 
des individus et compromettre leur carrière. À titre d'exemple, les médias traditionnels ont publié des histoires qui racontent de quelle manière de jeunes adultes ont été licenciés à la suite de certaines informations qu'ils ont publiées sur Facebook ${ }^{2}$. Dans la plupart des cas, c'est un individu se trouvant dans leurs cercles sociaux sur le RSN qui a communiqué ces informations à leur employeur.

2 Les jeunes adultes sont de plus en plus nombreux à faire usage de ces sites (Pew Research Center, 2013) ${ }^{3}$ qui pratiquent par défaut la surveillance. Plusieurs recherches menées en Amérique du Nord et en Europe se sont intéressées à leurs usages et les significations qu'ils y revêtent pour eux (Donath, 2007 ; Coutant et Stenger, 2010), alors que la question du non-usage des médias sociaux demeure peu explorée (Baumer et al., 2013). À l'ère où les individus sont de plus en plus branchés simultanément à plusieurs réseaux de communication (Jauréguiberry et Proulx, 2011; Rainie et Wellman, 2012), il est d'une pertinence sociale de comprendre les situations de non-usage volontaire, car elles nous permettent de saisir les raisons pour lesquelles les individus n'utilisent pas ou plus ces réseaux et les conséquences qui émergent quand l'on n'y est pas intégré.

Ce texte propose une analyse des logiques d'action liées aux usages, à la déconnexion et à la reconnexion au site. Il vise à mettre en lumière la déconnexion temporaire. La déconnexion illustre des conduites de préservation qui sont déployées pour prendre $d u$ recul et un temps à soi, face au trop-plein informationnel, aux sollicitations non souhaitées et à certaines situations où la vie privée est mise en péril. Il s'agit des formes de déconnexion privées. Ces jeunes adultes succombent à des pressions sociales par peur de manquer quelque chose et d'être exclus de certaines activités sociales et, pour en obtenir des bénéfices, ils se reconnectent à Facebook. Certaines logiques d'action liées à la reconnexion révèlent que les bénéfices associés aux pratiques de socialisation priment sur le besoin de se protéger de la surveillance. Ils intériorisent doucement le contrôle social. De ce fait, il est judicieux de se demander s'il est possible de vivre sans Facebook aujourd'hui et dans quelle mesure la faible visibilité des pratiques de surveillance institutionnelle et les changements constants introduits dans les architectures de certains médias sociaux façonnent les inégalités d'usages. Ce qui pourrait expliquer la déconnexion même temporaire.

\section{Les notions d'usage, de non-usage et de déconnexion volontaire}

4 L'usage d'une technologie engage une relation complexe et constante entre les choix que font les usagers et les propositions de l'objet technique (Proulx, 2005). Il prend toujours sens par rapport à un contexte social, étant à la fois médiatisé par la technique et le corps social (Jouët, 1993).

Selon Jauréguiberry et Proulx (2011, p. 106), l'étude des usages des technologies de l'information et de la communication (TIC) comme expérience a mis en évidence trois logiques d'action, notamment une logique d'intégration, une logique utilitaire et une logique critique. La première réfère à la volonté des individus de se connecter aux autres à travers les TIC, de ce fait, d'être intégré aux réseaux de communication. Dans ce processus, il s'agit de se construire un sentiment d'appartenance à un cercle social, selon ses besoins, ses goûts ou ses propres intérêts. La deuxième logique traduit la performance de l'individu quand il fait usage des technologies, par exemple la performance identitaire 
sur les sites de RSN dans un objectif de reconnaissance. Les travaux de Goffman (1973) sont souvent utilisés pour mettre en évidence la mise en scène de l'identité et les stratégies qui sont mobilisées pour protéger son image sociale et préserver celle d'autrui au cours des interactions. Dans certains cas, cette performance revêt un caractère instrumental, car il est question de repérer les membres du réseau, qui seraient enclins à apporter des bénéfices aux usagers. La troisième reflète la capacité de l'individu branché à avoir une réflexion critique par rapport à son expérience d'usager. Il peut prendre une distance par rapport à l'objet technique. Ceci lui permet de mieux gérer son temps ou d'opérer un retour sur soi loin de la technologie. Nous pouvons ici faire un lien avec les travaux de Giddens (1984). Le sociologue a soutenu que l'individu développe une réflexion dans le cours de l'action en mettant des mots sur des choses (conscience discursive) et en ajustant sa conduite quand il fait face à des changements dans certaines situations (conscience pratique). Il va veiller à maintenir un état de sécurité ontologique (confiance de base) dans le cours des actions afin de préserver une certaine autonomie. À partir des travaux de ces chercheurs, nous réalisons que l'étude des usages comme expérience permet entre autres de prendre en compte que des situations d'usages et de non-usages aux TIC peuvent s'inscrire dans la trajectoire des usagers.

6 L'une des premières formes de non-usage d'une technologie se reflète à travers la catégorie des usagers réfractaires que $\mathrm{E}$. Rogers avait identifiée en proposant sa « théorie de la diffusion " (Jauréguiberry, 2010). Le non-usager est ainsi considéré comme étant incapable d'adopter une innovation, alors que cette dernière revêt un aspect favorable. D'après Jauréguiberry, le non-usage a été abordé de façon implicite au cours des études qui traitent de la fracture numérique. Les premières études à ce sujet ont soulevé les problématiques liées à l'accès à la technologie, alors que celles qui ont été réalisées au cours des années 2000 traitent des modes d'utilisation et des inégalités d'usages. Des disparités en termes de capacités cognitives et de capital culturel, économique et social expliquent le taux de connexion aux TIC (ibid., pp. 26-27). L'une des premières études qui a souligné la dimension volontaire du non-usage est celle de S. Wyatt (1999), qui a argumenté que les non-utilisateurs doivent être abordés comme un groupe social en soi. L'auteure a identifié quatre catégories de non-usagers : 1 . ceux qui n'ont jamais utilisé Internet parce qu'ils n'en ressentent pas le besoin ; 2. ceux qui n'ont jamais utilisé Internet car ils n'ont pas accès au réseau pour diverses raisons ; 3. ceux qui ont arrêté de l'utiliser de manière volontaire; 4 . ceux qui ont dû arrêter de l'utiliser pour des raisons de coûts, de perte d'accès, etc. Il peut y avoir une articulation entre l'usage d'une technologie et le non-usage d'autres technologies. Certaines études antérieures à ce sujet ont montré que la porosité entre usages et non-usages d'Internet peut être façonnée par des inégalités économiques, culturelles et sociales (SIAD, 2009).

7 Jauréguiberry $(2010,2014)$ introduit une autre manière de concevoir les non-usages volontaires, qui se pose comme un choix que privilégient certains individus parfaitement équipés et connectés à Internet, qui subissent les effets négatifs liés à une hyperconnexion aux TIC. Le désir de se déconnecter rythme souvent avec celui de fuir «dans des situations [...] de trop-plein informationnel, de débordement cognitif, de harcèlement ou de surveillance dans lesquelles l'individu se sent dépassé ou soumis » (Jauréguiberry, 2014, p. 30). La déconnexion volontaire aux TIC est abordée comme étant partielle. Il ne s'agit pas de renoncer aux technologies, mais de maîtriser les usages. La déconnexion peut être une expérience forte que vit l'individu. Jauréguiberry qualifie cette dernière de déconnexion privée : «Elle est alors toujours volontaire et proactive. La 
déconnexion est dans ce cas souvent expliquée par la défense d'un temps à soi dans un contexte de mise en synchronie généralisée, par la préservation de ses propres rythmes dans un monde poussant à l'accélération, par le droit de ne pas être dérangé dans un environnement télécommunicationnel intrusif et par la volonté d'être tout à ce que l'on fait dans un entourage portant au zapping et à la dispersion » (ibid., p. 38).

Le chercheur reconnaît ainsi le rôle des technologies à submerger les individus d'informations, de sollicitations, qu'ils se sentent obligés de traiter. Cette obligation peut être imposée par son milieu professionnel ou par la famille et les amis. Ainsi, Lachance (2014) a discuté des obligations qui rendent la déconnexion aux TIC difficile pour les voyageurs. Il a souligné que, dans un contexte généralisé d'insécurité, les voyageurs se sentent de plus en plus obligés de donner des nouvelles à leurs proches. Les participants de cette étude ont essayé de contrôler l'intensité de leurs relations à travers la maitrise de leurs temps de connexion aux TIC pendant leurs voyages (ibid., p. 68). Ils ont instauré des plages horaires où ils sont susceptibles de téléphoner à leurs proches afin de les rassurer et de partager leurs expériences de voyage sans que cela empiète sur leurs vacances. La déconnexion aux TIC s'avère partielle et négociée.

9 À travers la notion de déconnexion volontaire, le non-usage apparaît comme étant partiel, voire temporaire. Cette notion déplace l'attention de la problématique de l'accès vers celle de l'excès (Jauréguiberry, 2010). Elle se révèle pertinente pour étudier les situations où l'usage de Facebook détient une place importante dans le quotidien de certains individus et montre que ces derniers optent pour une mise à distance temporaire de l'outil en vue de se protéger des effets négatifs liés à une hyperconnexion.

\section{Les usages de Facebook}

10 Depuis la création de Facebook, plusieurs recherches ont documenté les usages qui en sont faits. Des chercheurs ont mis en évidence que ce réseau social est utilisé par des usagers pour décliner certains aspects de leurs identités et que ce processus détient une dimension stratégique (Coutant et Stenger, 2010; Lardellier et Bryon-Portet, 2010). Facebook se positionne comme le support de la sociabilité ordinaire des étudiants américains (Donath, 2007; Rainie et Wellman, 2012). L'usage du site est également lié à des fonctions de surveillance (Proulx et Kwok Choon, 2011; Marwick, 2012). Par ailleurs, des études se sont penchées sur les usages de Facebook dans le cadre de certaines crises et des manifestations citoyennes (Kwok Choon, 2014). On discute également de l'incursion des professionnels tels que les politiciens et les journalistes au sein de ces sites (Newman, 2009). Dès son lancement, les groupes Facebook ont connu une popularité auprès des étudiants américains. Certaines recherches se sont intéressées aux bénéfices que peut apporter l'usage du site dans le contexte académique (De Villiers, 2010).

\section{Les formes de déconnexion à Facebook}

11 Les formes de déconnexion à Facebook sont liées plus particulièrement à une prise de conscience par rapport aux problèmes que pose le site en matière de protection de la vie privée. L'initiative Quit Facebook Day prise par plus de 41000 utilisateurs en 2010 a illustré la volonté d'un nombre notable d'utilisateurs de se déconnecter définitivement de Facebook. Un site $\mathrm{Web}^{4}$ a été créé afin de souligner la responsabilité du site quant à certains problèmes liés à la vie privée. On y a encouragé les utilisateurs de Facebook à 
rejoindre des sites alternatifs tels que Diaspora. Certains blogueurs ont aussi quitté Facebook car ils sont inquiets pour leur vie privée. Ils estiment que les propriétaires du site ne sont pas très transparents en ce qui concerne les procédés de récolte des données personnelles. D'autres ont affirmé qu'un trop grand investissement personnel est requis de leur part s'ils veulent susciter des émotions chez leurs amis Facebook. L'impression de s'engager dans un labeur gratuit en endossant certains produits culturels sur Facebook à travers l'activation du bouton "j'aime" a été une autre raison qui a mené à se déconnecter du site (Gehl, 2013, pp. 230-232). Des chercheurs (Baumer et al., 2013) ont mené des enquêtes quantitatives et qualitatives auprès des utilisateurs de Facebook qui ont quitté le site. Les facteurs liés à la déconnexion sont nombreux : protection de sa vie privée, prise de conscience quant à la dépendance à l'outil de communication et au fait de passer trop de temps sur le site. Ces chercheurs se sont intéressés plus particulièrement à ceux qui ont quitté définitivement le site, ceux qui ont limité leurs usages et aux utilisateurs qui ont évoqué le désir de se déconnecter pour plusieurs raisons, mais qui ne sont pas passés à l'action. Ils ont qualifié ce comportement de résistance latente (lagging resistance). Les motivations liées à la déconnexion temporaire sont évoquées; cependant, ces chercheurs ne traitent pas des logiques d'action qui sont liées à la reconnexion au site 5 .

\section{Méthodologie}

Notre recherche participe à un renouvellement par rapport aux études sur les non-usages des TIC, car elle permet de comprendre la déconnexion temporaire en s'intéressant aux logiques d'action liées aux usages, à la déconnexion et à la reconnexion à Facebook. Cette recherche a été effectuée dans le cadre d'une thèse de doctorat que nous avons menée sur les usages de différents réseaux sociaux numériques par les jeunes adultes québécois et leurs attitudes liées à la vie privée au sein de ces contextes en ligne ${ }^{6}$. De ce fait, le recrutement des jeunes adultes s'est basé sur deux critères : le simple fait d'utiliser ces sites et l'âge (18-34 ans). Les jeunes adultes sont ceux qui utilisent le plus les réseaux sociaux numériques dans la province du Québec. Le Cefrio (2011), qui mène des recherches quantitatives sur l'utilisation du numérique dans la province, a identifié les principales utilisations des médias sociaux par les jeunes Québécois: l'entretien d'un profil (par exemple sur Facebook, Linkedln, MySpace ou encore Twitter), le relais de l'information, la création du contenu. En sondant 561 internautes, il en ressort que les 18-24 ans passent en moyenne 8,6 heures par semaine sur les médias sociaux. D'après le même rapport, dans la population des jeunes adultes qui utilisent les RSN dans la province, la majorité d'entre eux sont des étudiants.

L'appel à participation à la recherche a été envoyé à 33 étudiants inscrits à un cours au baccalauréat d'une université au Québec par courriel. En prenant en compte le critère de saturation, nous avons décidé de nous entretenir avec 20 d'entre eux. Nous sommes devenus leurs amis sur Facebook, après qu'ils ont signé le formulaire de consentement ${ }^{7}$. Les entrevues qualitatives non dirigées ont été menées en explorant simultanément les profils Facebook de ces jeunes adultes. Elles ont pris la forme d'une discussion qui a duré 90 minutes, pendant laquelle nous avons posé à chaque participant cette question ouverte : « Peux-tu me montrer ce que tu fais sur Facebook? » Il s'agissait de laisser libre cours au récit de la personne interviewée, en la relançant de temps en temps, dans le but de faciliter le développement de certaines idées (Bonneville, 2007 ; Livingstone, 2008). 
Notre démarche était ethnographique; de ce fait, nous avons voulu comprendre les usages à partir des discours des usagers. Pour reprendre les propos de Spradley (1979, p.93, traduction de l'anglais): «Nous voulons précisément éviter d'imposer des catégories provenant de l'extérieur qui visent à définir les tendances de manière ordonnée; nous voulons plutôt favoriser leur découverte. $»^{8}$ À notre connaissance, il n'existe pas d'étude qualitative qui offre une description approfondie des usages de Facebook par les jeunes adultes québécois et de la déconnexion temporaire au site.

Pendant ces entretiens qui se sont déroulés entre les mois de mars et de mai 2013, certains des usagers ont évoqué leurs situations de déconnexion à Facebook. Le questionnement de recherche suivant a émergé au cours de l'étude de terrain : comment décrire les logiques d'action qui sont liées aux usages de Facebook, à la déconnexion et à la reconnexion au site? Au cours de l'année 2014, nous nous sommes engagée dans des conversations informelles, par le biais de la messagerie Facebook, avec les participants qui ont évoqué le thème de la déconnexion lors des entretiens, afin d'approfondir notre compréhension des raisons qui avaient conduit à cette déconnexion temporaire, puis à la reconnexion. Des pseudonymes ont été utilisés pour citer les propos de ces participants dans un objectif de protéger leur vie privée. Les entrevues ont été retranscrites et le codage des données a été effectué manuellement en identifiant les liens sémantiques entre les propos des différents usagers et les thèmes auxquels ils renvoient, ainsi que les relations entre les différents thèmes (ibid.). Les sections suivantes proposent les principaux résultats issus de cette recherche.

\section{L'usage de Facebook dans le contexte des loisirs et dans le milieu académique}

15 Ces jeunes adultes utilisent Facebook à la fois par le biais de leur téléphone mobile et de leur ordinateur. Ils font usage du site plusieurs fois par jour sur leur lieu de travail, quand ils sont en salle de classe et lors de leurs différents déplacements. Ces usagers ont entre 100 et 600 amis Facebook ${ }^{9}$. Ils comptent dix amis plus au moins proches dans leur réseau, cinq membres de leur famille; le reste de leurs cercles sociaux est composé de connaissances. Ces dernières sont des personnes côtoyées au moins une fois quand ils étaient au secondaire et au cégep ainsi que d'anciens collègues avec lesquels ils ne communiquent plus. La majorité des usagers ont avoué garder ces connaissances dans leurs cercles sociaux pour d'éventuels bénéfices qu'ils pourraient générer de ces liens faibles.

\section{La surveillance sociale}

Leur premier réflexe en accédant au site est de vérifier les notifications et leur fil d'actualité ${ }^{10}$. Ils le survolent plusieurs fois par jour sans pour autant contribuer aux conversations et observent les profils de leurs amis Facebook. Par exemple, Corinne avoue qu'elle consacre 15 minutes pour regarder son fil d'actualité et consulter les profils de ses amis quotidiennement, alors que Sébastien passe son temps libre à observer les nouvelles photos publiées par ses amis. Il s'agit également de scruter les événements organisés par leurs amis Facebook. Le site est ainsi utilisé pour s'engager dans des recherches sociales. En pratiquant cette activité, les usagers accumulent des connaissances sur leurs cercles sociaux en ligne (Marwick, 2012). En même temps que ces 
utilisateurs pratiquent cette forme de surveillance, ils se soumettent aussi au regard des autres en dévoilant leurs informations personnelles. Force est alors de constater que certains usagers se sont sentis «traqués » par d'autres utilisateurs, tels que leurs expartenaires amoureux sur Facebook.

\section{La mise à jour du statut}

La mise à jour du statut Facebook consiste principalement à s'exprimer sur un sujet par le biais de la page de son profil en faisant usage de divers contenus médiatiques (textes, photos, vidéos, etc.). Nous avons identifié trois formes de mise à jour du statut. Premièrement, le partage des selfies ${ }^{11}$ et des photos qui les illustrent avec leurs amis ou des membres de leur famille lors de leurs différentes activités quotidiennes ou d'événements (fête, vacances, cérémonie de remise de diplôme, activités en plein air, etc.). L'application mobile Instagram est utilisée pour ajouter des filtres aux photos avant qu'elles soient publiées sur Facebook. Les usagers ne partagent pas les photos sur lesquelles ils semblent être à leur désavantage physiquement. Les photos qui montrent ainsi l'utilisateur à différents événements en présence de ses amis ou des membres de la famille sont partagées dans une volonté de montrer qu'il a une vie sociale riche. Le paraitre occupe ainsi une place importante dans la construction du profil. Les usagers montrent une volonté de construire une image flatteuse d'eux-mêmes (Lardellier et Portet, 2010).

Deuxièmement, les insides sont une manière subtile de partager une information sans pour autant dévoiler sur Facebook les conversations qui se sont déroulées et l'événement qui a eu lieu, et qui ont conduit à la création du statut. Ils permettent de "faire signe » aux amis proches et tendent à mettre en scène des blagues qui ont été faites dans un contexte hors ligne. Ils s'apparentent à des activités de l'ordre du toilettage social (Donath, 2007). C'est un moyen privilégié par ces utilisateurs d'entretenir au quotidien des liens sociaux proches par le biais de Facebook. Par exemple, Ludovic explique cet inside :

" C'est une joke de bord. C'est un peu vulgaire, c'est ça (rires). Personne ne comprend, à part Mika qui était là au bar. Elle étudie en sexologie, puis, au bar, elle a dit “T'as-tu déjà fait ça, Ludovic ?" Et puis, là, elle dit : "La prochaine fois que tu me verras, tu me diras si tu l'as faite. Puis, à chaque fois que tu vas me voir, il faudra que tu me le dises." C'est pour ça qu'elle dit : "Puis, l'as-tu faite ?" Et je dis non. Ça ressemble pas mal à ça (rires). » (sic)

La troisième forme de mise à jour des statuts consiste à informer ses «amis » Facebook des sujets d'actualité en publiant des liens, des photos, des extraits d'articles, etc. Par exemple, Mira est frustrée car elle ne comprend pas pourquoi des personnes sont contre le mariage homosexuel. Ainsi, elle a publié un article à ce sujet, alors que Noémie a partagé un article sur la starisation des meurtriers afin de faire réagir ses amis Facebook. Le partage des nouvelles est rendu possible sur Facebook grâce à son interface qui propose des fonctionnalités telles que le fil d'actualité et la possibilité d'intégrer des liens provenant des différents sites de nouvelles et d'autres médias sociaux tels que YouTube (Newman, 2009). À travers la publication des sujets d'actualité sur Facebook, ces jeunes adultes montrent une certaine volonté de sensibiliser leurs cercles sociaux en ligne sur des problèmes qui traversent notre société grâce aux différents contenus médiatiques. 


\section{Contribuer aux conversations en lien avec les travaux universitaires} en groupe, ils ont pour réflexe de suggérer la création d'un groupe Facebook. Ils partent du principe que leurs collègues ont un compte Facebook en regard de la popularité du site. Les contenus qui sont partagés ont pour finalité de faire avancer le travail de rédaction et organiser des rencontres en face-à-face. Sheila et Lily se sont ainsi exprimées sur les bénéfices liés à l'usage de Facebook dans le cadre de leurs travaux universitaires. Sheila :

"Ce qu'on aime avec Facebook c'est que tu peux commenter sur le travail et tout le monde peut le voir. Dans le fond, c'est pratique dans le sens où il est possible de partager plusieurs fichiers. [...] Oui, dans le fond, le groupe de Facebook de recherche servait uniquement à communiquer entre nos rencontres. On utilisait le mur pour nous faire des petits rappels : “Oh, ne l'oublie pas! On a des rencontres à telle date et à telle heure !" » (sic)

Lily : « Par exemple, sur le groupe "mouvements sociaux". Genre, on peut voir que j'écrivais souvent. Parce qu'on n'avait pas beaucoup l'occasion de se voir souvent, on se rencontrait au début et on a un peu divisé le travail. J'ai pris la grosse partie du travail et je vais vous tenir au courant. Ça, ce sont les questions d'entrevues. On s'encourage, pour se rencontrer. On planifie les rencontres. Du début à la fin, on interagit dessus. » (sic)

Le site Facebook se positionne pour les étudiants dans le contexte académique comme un outil qui permet les conversations informelles en lien avec des travaux universitaires et la collaboration autour d'un projet spécifique (de Villiers, 2010). Si l'un des membres du groupe n'utilise pas Facebook, l'information lui sera acheminée par courriel. Dans la plupart des cas, les étudiants encourageront ce collègue à créer un profil Facebook.

\section{Les logiques d'action d'intégration et utilitaire}

Dans le processus d'usage, les utilisateurs projettent différents aspects de leur identité, tel un « moi » soucieux des problèmes qui traversent notre société, qui jouit des attributs physiques avantageux, ainsi qu'une vie sociale riche et qui accorde de l'importance aux amis proches ou à ce que leurs coéquipiers ont partagé sur les groupes Facebook. Leurs usages reflètent une centration sur le « moi » et sur la manière dont ce « moi » sera perçu par autrui. Ils confèrent ainsi différentes impressions à l'audience en présence, composée des individus avec lesquels ils entretiennent des liens sociaux forts et des liens faibles d'ordre latent. La majorité des usagers ont avoué être très heureux quand leurs billets génèrent un grand nombre de «j'aime ». Il s'agit là d'avoir le sentiment de participer à l'existence d'une communauté en ligne et de se mettre en scène dans l'optique d'obtenir des bénéfices sociaux, émotionnels et liés au rendement. Par exemple, Élissa, Noémie et Sébastien ont affirmé, que grâce à ces contacts, ils ont accès à un éventail d'informations lié aux événements. Ces amis Facebook pourraient également leur être utiles dans la recherche d'un emploi ou d'informations pertinentes en relation avec leur domaine d'activité. Dans l'ensemble, leurs usages reflètent les logiques d'action utilitaire et d'intégration. Pour reprendre les propos de Jauréguiberry et Proulx (2011, p. 107, p. 110) à propos de la logique d'intégration et utilitaire, 
« l'intégration passe par la connexion. [...] D’un côté, les « amis », connaissances et profils sont triés selon un ensemble de critères permettant à l'internaute de mesurer leur plus ou moins grande capacité à répondre favorablement à ces attentes. Il s'agit de repérer, évaluer et cataloguer les membres du réseau selon une logique purement instrumentale : qui peut m'amener quoi et dans quelle condition?»

\section{Les formes de déconnexions temporaires d'ordre privé}

La majorité des jeunes adultes ont réfléchi sur la relation qu'ils entretiennent avec le site Facebook. Plusieurs d'entre eux sont conscients de la grande place que détient l'usage du RSN dans leur quotidien et ont avoué que quitter le site serait inimaginable. Ils se considèrent comme étant "dépendants " au RSN. Certains ont tenté l'expérience de la déconnexion, mais elle a été de courte durée, entre 5 et 14 jours. Il s'agit des formes de déconnexion privées qui ont pour objectif de regagner une forme de contrôle sur leurs usages et de se préserver (Jauréguiberry, 2014). La déconnexion se vit comme une expérience forte où les usagers ressentent le besoin de s'éloigner du site.

\section{Le besoin de faire une pause et de fuir des sollicitations non souhaitées}

Certains usagers prennent conscience du temps qu'ils investissent à mettre en scène leur identité et à scruter les activités de leurs amis Facebook. Par exemple, Mira a reconnu qu'un usage intensif peut avoir des impacts négatifs sur la productivité académique :

"Oui. J'ai même par deux fois demandé à mon chum de changer mon mot de passe deux fois pour ne plus y aller, deux semaines pour voir ce que ça faisait et aussi pour m'empêcher de mettre mes travaux en péril. Voir si j'étais capable de vivre sans [...] parce que chaque fois qu'on est concentré ou pas sur nos affaires il y a toujours des trucs à voir sur Facebook, alors on a toujours envie d'ouvrir une page de plus et de faire une pause là, mais la pause ne finit plus et on perd notre temps. » (sic)

Le fait de quitter Facebook permet à ces usagers de prendre du recul par rapport au site, de se retrouver et d'investir leur temps dans l'accomplissement des tâches prioritaires. À ce stade, ils considèrent que la déconnexion du site aura un impact positif sur leur productivité académique. La déconnexion renvoie à vouloir préserver un temps pour soi et à un besoin de s'éloigner des sollicitations constantes médiatisées par la technique (Jauréguiberry, 2014). Par exemple, un trop-plein de notifications sur Facebook.

Dans certains cas, l'individu opte pour la déconnexion afin de fuir certaines sollicitations à participer aux représentations que donnent les autres au cours des interactions ou à se conformer à certaines d'entre elles. Par exemple, Noémie et Élissa ont évoqué les raisons pour lesquelles elles ont décidé de quitter le site. Noémie :

« Tout cela est bien artificiel. J'avais cette impression que tout le monde se mettait en scène, publiait une image bonifiée de lui-même. À dire vrai, ça me faisait de la peine un peu quelquefois, car j'avais l'impression que ma vie était un peu beige en comparaison. » (sic).

Élissa : « Oui, j'avais essayé de supprimer mon compte pendant cinq jours, tout était comme neuf, il n'y avait pas de problème. Cela faisait vraiment longtemps que j'y pensais. 
Je pensais qu'on pouvait vivre sans Facebook. C'est juste à ce moment qu'il y a eu un conflit avec l'une de mes amies, ce n'est pas très grave, mais c'est le genre d'amie qui va mettre ses états d'âme sur Facebook. "Oh, mon dieu, je me suis fait de la peine pour ma meilleure amie." Ça ne me tente pas. Je vais essayer de voir comment ça fait de ne pas avoir Facebook et de ne pas voir ses états d'âme douloureux et suicidaires sur Facebook. » (sic)

Ces utilisateurs se protègent de certains préjudices émotionnels que peuvent causer des formes d'humiliation à la suite d'une situation de conflit avec un ami Facebook ou le fait de se conformer à la norme sociale du paraître. Comme Jauréguiberry et Proulx (2011, p.121) l'ont mentionné, les différentes mises en scène à travers les réseaux de télécommunications se construisent autour de différents critères précis. Présenter une belle image de sa personne et déverser certains états d'âme peuvent être des critères. Dans le premier cas, des individus peuvent vivre cela comme une forme de conformisme qui les lie étroitement à leurs déterminants physiques et, par crainte de ne plus s'appartenir, quittent le réseau social. Dans le deuxième cas, il s'agit d'échapper au rôle social suivant : être un ami à l'écoute qui offre une forme de soutien en ligne, quand on est soi-même le sujet du problème en question.

\section{La protection de la vie privée} puissent tracer ses déplacements par le biais du site et avoir accès à ses informations :

"J'ai quitté Facebook le printemps passé pour protéger mon identité. Mon ancien copain avait eu un différend avec un autre gars et on pensait qu'il m'avait retrouvé et qu'il voulait me causer des problèmes puisque j'avais reçu des demandes d'ami de personnes ayant de faux profils (seulement deux amis, pas d'historique sur la timeline), donc j'ai fermé mon compte pour une semaine le temps que ça se calme. J'avais peur qu'on puisse me tracer puisque je m'indique à des lieux parfois, je publie beaucoup de photos. Ensuite, Facebook me manquait trop, donc j'ai décidé de prendre le risque de le rouvrir. » (sic)

De son côté, Joey a décidé de quitter le site parce qu'il s'est senti traqué par une expartenaire amoureuse :

«Il y avait une fille que je fréquentais à l'époque qui avait été chercher des informations sur mon Facebook, mais des informations que je ne savais même pas qu'elles étaient là, des informations par rapport à ma vie privée. Elle s'est rendue compte de ces trucs-là. Elle est revenue sur moi avec ça, ça a comme brisé un lien de confiance. Je me suis rendu compte que je ne pouvais pas vraiment lui en vouloir parce que c'est l'information que j'affichais publiquement. Je me rendais compte que Facebook pouvait être un outil à double tranchant, j'ai décidé de tirer un trait sur Facebook, puis je suis revenu là-dessus pour les travaux de groupes. Je pense que mon profil est assez sobre. » (sic)

La déconnexion s'effectue ainsi à la suite d'un sentiment de perte de contrôle de la vie privée. En comparaison avec les études antérieures traitant de ce sujet, ici les individus ne remettent pas en cause les pratiques de surveillance institutionnelle, mais plutôt les pratiques de surveillance sociale qui sont médiatisées par le RSN (Gehl, 2013; Baumer et al., 2013). 


\section{La reconnexion au site : Le FOMO, les pressions sociales, la crainte d'être exclu de certaines activités sociales et l'obtention des bénéfices}

31 Au moment de se déconnecter, ces jeunes adultes ont la conviction qu'ils peuvent « vivre sans Facebook». Cependant, la reconnexion au site s'effectue car ils craignent de manquer quelque chose. Comme l'ont souligné Rainie et Wellman (2012, p. 159), le FOMO est présent quand les réseaux d'amis sont dispersés géographiquement et qu'un seul outil de communication est privilégié pour entretenir ces différentes relations sociales. Il est difficile de maintenir le contact avec ce grand nombre d'individus au sein d'un contexte physique et par téléphone. Ceci s'applique aux usages de ces jeunes. Facebook est utilisé comme outil principal pour entretenir des liens forts et des liens faibles d'ordre latent. Le sentiment de manquer quelque chose prend corps quand leurs amis se rendent compte de leur absence du site, les incitent à s'y reconnecter et leur racontent les événements qu'ils ont manqués en ligne. Par exemple, Noémie a expliqué que son amie Pippa l'a encouragée à se reconnecter sur Facebook. C'est pour cette raison qu'elle a rédigé cet inside :

«Je lâche prise, Pippa. T’es le démon!»

Sheila a admis que ses amis l'ont contactée lorsqu'elle a quitté le site pour lui dire que Marc qui est un ami éloigné avait dévoilé sur Facebook qu'il était célibataire. Elle avait le sentiment d'être "déconnectée des potins". La reconnexion montre que ces jeunes adultes succombent dans une certaine mesure à des pressions sociales. Lachance (2014, p.71) a discuté des pressions sociales qu'expérimentent les voyageurs à demeurer connectés aux TIC :

«L'idéal de la déconnexion totale est rudement mis à l'épreuve. Les pressions sont importantes, les arguments de l'entourage s'imposent, implacables. »

Dans ce cas comme dans l'autre, c'est la qualité des relations sociales qui est en jeu si la déconnexion aux TIC s'avère longue. Si ces relations sociales ne sont pas entretenues à travers Facebook, ils risquent d'être exclus de certaines activités sociales. Par exemple, Sheila et Mira ont mentionné qu'il est nécessaire d'avoir accès aux annonces de certains événements publiés sur Facebook pour pouvoir s'y rendre, tandis que Joey et Noémie ont avoué que participer aux conversations en lien avec les travaux universitaires sur Facebook est primordial s'ils veulent les remettre dans les délais escomptés. Comme mentionné précédemment, ils peuvent également imposer subtilement l'injonction de la connexion à leurs collègues qui n'ont pas un profil sur Facebook, avec pour objectif d'optimiser les discussions en lien avec les travaux universitaires.

Les RSN se positionnent comme des «boutiques relationnelles " permettant aux usagers de créer des ponts entre des réseaux d'usagers qu'ils côtoient dans un contexte en ligne et hors ligne pour obtenir du soutien et d'autres types de bénéfices (Rainie et Wellman, 2012). De ce fait, quitter le site définitivement se révèle une tâche difficile et préjudiciable pour leur vie sociale. 


\section{De la déconnexion à la reconnexion}

\section{Entre la protection de l'autonomie d'action et la recherche d'un compromis}

Les formes de déconnexion observées sont des conduites de préservation qui visent à protéger l'autonomie d'action et à établir une distance réflexive (Jauréguiberry et Proulx, 2011, p. 118), en présence du trop-plein informationnel, des sollicitations non souhaitées et des situations où la vie privée est mise en péril. Ce sont des situations jugées critiques pour le «moi ». Giddens (1984, p. 112) a expliqué les conséquences liées à ces situations : «La perturbation des routines ordinaires de la vie et les attaques délibérées et constantes qui leur sont portées engendrent un haut degré d'angoisse ainsi qu'un "dépouillement" des réponses socialement acceptées et associées à la sécurité du contrôle du corps ainsi qu'à un cadre familier et prévisible de vie sociale. » Par conséquent, l'éloignement du site permet de se tenir à distance des situations jugées critiques, de regagner une forme de contrôle sur le cours des actions et de réfléchir sur les actions futures. La déconnexion est ainsi liée à la logique d'action critique.

En se reconnectant au site, ces jeunes adultes essaient de trouver un juste milieu entre l'emploi de Facebook pour des raisons utilitaires et sociales et le besoin de se préserver. Par exemple, certains participants ont admis qu'en revenant sur Facebook ils ont adopté un profil plus «sobre ». D'autres usagers évitent de s'identifier sur des photos pour se protéger de la surveillance sociale ou ont décidé de ne plus porter attention aux sollicitations non souhaitées provenant de certains amis Facebook. Pour reprendre les propos de Jauréguiberry et Proulx (2011, p. 118) :

« la nécessité de maximaliser ses opportunités de gain, de rentabiliser son temps, d'augmenter l'efficacité de ses contacts, et, de l'autre, la volonté de "ne pas perdre son âme dans cette course", d'avoir un rapport autre que purement instrumental avec les autres, de préserver une distance synonyme d'autonomie individuelle, se traduit par une recherche de compromis visant à conjuguer au mieux l'une de ces exigences avec l'autre. »

\section{Une connaissance limitée des modes opératoires des RSN et l'intériorisation douce du contrôle social}

Quand ils se reconnectent à Facebook, les jeunes adultes ont le sentiment de maîtriser leurs situations d'usage ; cependant, cette autonomie se retrouve court-circuitée par leurs connaissances limitées en matière de procédés de surveillance institutionnelle, qui façonnent leurs expériences d'usage (Proulx et Kwok Choon, 2011, p. 110). Ainsi, leurs informations personnelles se sont retrouvées exposées, car ils n'étaient pas au courant des différents changements survenus dans les conditions de service de Facebook et les politiques de confidentialité. D'ailleurs, la majorité d'entre eux ont avoué ne pas avoir aperçu l'annonce de ces changements en contexte.

Bien que ces jeunes adultes aient activé les paramètres de confidentialité «amis seulement ", ils n'ont pas consulté cette section depuis l'intégration de la timeline ${ }^{12}$. En explorant ces paramètres durant l'entretien, ils ont ainsi constaté que certaines de leurs informations personnelles étaient soumises à un public élargi par défaut. Par exemple, leurs profils étaient répertoriés par les moteurs de recherche et les photos de profil étaient visibles par le public. Ils n'étaient pas au courant que les applications peuvent 
avoir accès à certaines informations personnelles par le biais de leurs amis Facebook. Par ailleurs, en présence des sollicitations non souhaitées à participer aux représentations que donnent leurs amis Facebook et des situations de surveillance sociale, ces jeunes adultes auraient pu bloquer les usagers en question en activant les paramètres de confidentialité adéquats, mais ils ne connaissaient pas l'existence de ces paramètres. Force est alors de constater que la faible visibilité des pratiques de surveillance institutionnelle en contexte marginalise dans une certaine mesure la réflexivité des usagers. Si les changements apportés à ces pratiques ne sont pas annoncés de manière appropriée dans le contexte d'usage, les utilisateurs risquent de ne pas les voir. De ce fait, ils ne peuvent pas en prendre connaissance et entamer ainsi des actions réfléchies pour protéger leur vie privée.

Par ailleurs, la reconnexion montre qu'ils se soumettent volontairement à nouveau aux pratiques de surveillance sociale. Ces jeunes adultes sont conscients des risques liés à la surveillance sociale, comme Mira :

"Quand je publie des photos, je me dis, j'ai beaucoup de photos, quelqu'un pourrait m'écœurer, quelqu'un qui me suit, je ne sais pas. On se dit que ça n'arrive pas à moi et le jour que ça t'arrive c'est trop tard. » (sic) - ou Noémie - « À partir du moment que tu publies et qu'il y a un autre qui a accès ça ne t'appartient plus vraiment. Il faut que tu fasses attention, faire des captures d'écran, le partager dans un autre contexte, le bouger, le changer avec Photoshop, c'est facile là. » (sic)

Toutefois, ils prennent le risque d'exposer leurs informations personnelles devant une audience composée de gens qu'ils ne fréquentent plus dans un contexte en ligne et hors ligne pour en obtenir des bénéfices. Nous sommes en présence de ce que Proulx et Kwok Choon (2011) ont défini comme le second mouvement contradictoire lié aux RSN, notamment l'intériorisation douce du contrôle social ${ }^{13}$ par des usagers, bien qu'ils soient plus ou moins conscients de certains risques associés à une exposition de l'information personnelle dans un contexte de surveillance.

\section{Conclusion}

41 Cette recherche exploratoire a analysé les logiques d'action liées aux usages et à la déconnexion temporaire de Facebook. L'usage du site est ainsi lié à une logique utilitaire et d'intégration. La déconnexion au site illustre la logique d'action critique, à travers des conduites de préservation ayant pour objectif de défendre un temps à soi en présence d'un trop-plein informationnel, des sollicitations non souhaitées et des situations où la vie privée est mise en danger. Ces usagers ont peur de ne plus s'appartenir et de ne plus maîtriser leurs usages. La déconnexion est volontaire, partielle et se vit comme une expérience forte. L'analyse de la déconnexion temporaire a mis en évidence la connaissance limitée des usagers par rapport à certains modes opératoires du site. Ces jeunes adultes succombent aux pressions sociales et retournent sur le site par peur de manquer quelque chose et d'être exclus de certaines activités sociales et pour en obtenir des bénéfices. La reconnexion au site illustre la recherche d'un juste milieu entre le besoin de s'intégrer à leurs réseaux sociaux en ligne pour des raisons utilitaires et de se préserver. Elle met également en évidence une forme d'intériorisation douce du contrôle social. 

inégalités économiques, culturelles et sociales, qui sont des facteurs d'explication des inégalités d'usages. Dans le processus du réseautage, les usagers n'ont pas les mêmes capacités d'agir. La connaissance en matière de procédés de surveillance et de protection de la vie privée est un atout quand on est confronté à des situations de surveillance sociale ou à des sollicitations non souhaitées de la part de certains amis. Pour développer des compétences techniques, il est essentiel d'avoir une connaissance des modes opératoires de la technique. Notre hypothèse est que, dans le cadre des usagers des médias sociaux, il y a un lien étroit entre le savoir-faire des utilisateurs et la déconnexion aux médias sociaux. Des usagers qui ont un savoir-faire limité en regard d'une connaissance restreinte des modes opératoires de la technique seront plus enclins à se déconnecter des sites qu'ils utilisent s'ils sont confrontés à des situations problématiques en lien avec leurs usages. Nous notons par ailleurs que la faible visibilité des pratiques de surveillance institutionnelle en contexte ne facilite pas leur prise de conscience par les usagers. En conséquence, les utilisateurs n'arrivent pas à entreprendre des actions réfléchies pour protéger leur vie privée au cours des interactions sur les RSN.

Il est également judicieux de se demander dans quelle mesure la faible visibilité des pratiques de surveillance institutionnelle et les changements techniques constants effectués aux architectures des RSN façonneraient ainsi dans une certaine mesure les inégalités d'usage. Ce qui contribuerait au fait que les usagers ayant une certaine difficulté à s'adapter aux développements accélérés de ces technologies optent pour la déconnexion, même temporaire. Il est pertinent de se demander s'il est possible de vivre sans Facebook aujourd'hui. Ces usagers sont sujets à des pressions sociales à maintenir une présence dans ce contexte, en même temps qu'ils imposent subtilement l'injonction de la connexion à certains collègues. Par ailleurs, les bénéfices liés aux pratiques de socialisation priment sur le besoin de se protéger des conséquences négatives liées aux pratiques de surveillance. Par conséquent, nous devons nous demander dans quelle mesure le positionnement du RSN en tant qu'outil principal de socialisation et de maintien de liens majoritairement faibles concourrait au besoin permanent d'y demeurer connecté.

BAUMER E., ADAMS P., Khovanskaya V. D., Liao T. C., Smith M. E., Schwanda Sosik V. et Williams K., 2013, « Limiting, Leaving, and (re)Lapsing : An Exploration of Facebook Non-Use Practices and Experiences », in Proceedings of the SIGCHI Conference on Human Factors in Computing Systems, du 27 avril au 2 mai, Paris, http://ericbaumer.com/wp-content/uploads/2013/04/CHI2013-FBLL-13camera.pdf, dernière consultation le 8 octobre 2016.

BENNETT C. J., Haggerty K. D., Lyon D. et Steeves V., 2014, Vivre à nu : La Surveillance au Canada, Athabasca University Press.

BONNEVILLE L., GROSJEAN S. et LAGACÉ M., 2007, Introduction aux méthodes de recherche en communication, Montréal, Gaëtan Morin Éditeur, Chenelière Éducation.

CEFRIO, 2011, « L'Engouement pour les médias sociaux au Québec », NETendances, vol. 2, pp. $1-20$.

CHANDER A., 2012. « Facebookistan », North Carolina Law Review, vol. 90, pp. 1807-1842.

DE VILLIERS M. R., 2010, « Academic Use of a Group on Facebook : Initial Findings and Perceptions », in Proceedings of Informing Science \& IT Education Conference (InSITE), du 19 au 24 juin, Cassino (Italie), pp. 173-190, http://proceedings.informingscience.org/InSITE2010/ InSITE10p173-190Villiers742.pdf, dernière consultation le 31 mars 2016. 
DONATH J., 2007, « Signals in Social Supernets », Journal of Computer-Mediated Communication, vol. $13, \mathrm{n}^{\circ} 1$, pp. 231-251.

FACEBOOK, 2015, www.facebook.com, dernière consultation le 31 mars 2016.

GEHL R. W., 2013, « "Why I Left Facebook” : Stubbornly Refusing to Not Exist Even After Opting out of Mark Zuckerberg's Social Graph », in G. Lovink et M. Rasch, Unlike Us Reader : Social Media Monopolies and Their Alternatives, Amsterdam, Institute of Network Cultures, pp. 220-238.

GIDDENS A., 1984, La Constitution de la société : Éléments de la théorie de la structuration, Paris, PUF.

GOFFMAN E., 1973, La Mise en scène de la vie quotidienne, Paris, Les Éditions de Minuit.

JAURÉGUIBERRY F, 2014, «La Déconnexion aux technologies de communication », Réseaux, vol. 4, $\mathrm{n}^{\circ} 186$, pp. 15-49.

JAURÉGUIBERRY F. et Proulx S., 2011, Usages et enjeux des technologies de communication, Toulouse, Érès.

JAURÉGUIBERRY F., 2010, « Les Théories sur le non-usage des technologies », in Actes des travaux du comité de recherche "Sociologie de communication ", Colloque international " Dispositifs techniques de communication humaine : transformations du lien et nouveaux lieux sociaux », 19 et 20 mai, Namur (Belgique), pp. 22-31, http://web.univ-pau.fr/RECHERCHE/SET/AISLFCR33/ DOCS_SOCIO/2010/Actes_AISLF_CR33_Namur_2010.pdf, dernière consultation le 8 octobre 2016. JOUËT J., 1993, « Pratiques de communication et figures de la médiation », Réseaux, vol. 11, n 60, pp. 99-120.

KAPLAN A. M. et Haenlein M., 2010, « Users of the World, Unite! The Challenges and Opportunities of Social Media », Business Horizons, vol. 53, n 1, pp. 59-68.

KWOK CHOON M. J., 2014, « Coveillance, coproduction et codiffusion des nouvelles : les changements survenus dans la circulation de l'information à l'ère des réseaux sociaux numériques ", in Actes du colloque Concentration de la propriété des médias, changements technologiques et pluralisme de l'information, 26 et 27 septembre, Montréal, pp. 189-197, www.cricis.uqam.ca/IMG/pdf/CRICIS_Actes_Colloque_concentration_et_pluralisme_VF.pdf, dernière consultation le 8 octobre 2016.

KWOK CHOON M. J., et CARON I., 2012, «Artveillance practices : From Graffiti to Social Network Sites ", in TEM 2012 : Proceedings of the Technology \& Emerging Media Track, Canadian Communication Association, du 30 mai au 1er juin, Waterloo (Canada), www.tem.fl.ulaval.ca/ www/wp-content/PDF/Waterloo_2012/KWOK-CARON-TEM2012.pdf, dernière consultation le 31 mars 2016 .

LACHANCE J., 2014, « De la déconnexion partielle en voyage : L’Émergence du voyageur hypermoderne », Réseaux, vol. 4, n²186, pp. 51-76.

LARDELIER P. et Bryon-Portet C., 2010, «Ego 2.0. Quelques considérations théoriques sur l'identité et les relations à l'ère des réseaux ", Les Cahiers du numérique, vol. 6, n 1, pp. 13-34.

LYON, D. (dir.), 2001, Surveillance Society: Monitoring Everyday Life, Milton Keynes, Open University Press.

MARWICK A. E., 2012, « The Public Domain: Surveillance in Everyday Life », Surveillance \& Society, vol. $9, n^{\circ} 4$, pp. 378-393.

NEWMAN N., 2009, "The Rise of Social Media and Its Impact on Mainstream Journalism », Reuters Institute for the Study of Journalism, University of Oxford, www.sssup.it/ 
UploadDocs/6635_8_S_The_rise_of_Social_Media_and_its_Impact_on_mainstream_journalism_Newman_07.pdf , dernière consultation le 31 mars 2016.

PROULX, S., 2005, « Penser les usages des technologies de l'information et de la communication aujourd'hui : enjeux - modèles - tendances ", in Enjeux et usages des TIC : aspects sociaux et culturels, tome 1, sous la direction de L. VIEIRA et N. PINÈDE, Presses universitaires de Bordeaux, pp. 7-20.

PROULX S. et Kwok Choon M. J., 2011, «L'Usage des réseaux socionumériques : une intériorisation douce et progressive du contrôle social », Hermès, n 59, pp. 105-111.

Quit Facebook Day, www.quitfacebookday.com, dernière consultation le 31 mars 2016.

RAINIE L. et WELLMAN B. (dir.), 2012, Networked: The New Social Operating System, MIT Press.

RANDALL D. et RICHARDS V., 2008, « Facebook can ruin your life. And so can MySpace, Bebo... » The Independent, www.independent.co.uk/life-style/gadgets-and-tech/news/facebook-can-ruinyour-life-and-so-can\%\\%-myspace-bebo-780521.html, dernière consultation le 6 février 2015.

SIAD, 2009, « Portrait des non-internautes aquitains en 2009 », Aquitaine AnalyTIC, www.aecom.org/content/download/967/14308/version/1/file/Analytic6_noninternautes.pdf, dernière consultation le 31 mars 2016.

SPRADLEY J. P., 1979, The Ethnographic Interview, New York, Holt, Rinehart and Winston.

WYATT S., 1999, « They came, they surfed, they went back to the beach: why some people stop using the Internet », http://virtualsociety.sbs.ox.ac.uk/reports/surf.htm, dernière consultation le 31 mars 2016.

ZICKUHR K., 2013, « Who's Not Online and Why », Pew Research Center, www.pewinternet.org/2013/09/25/whos-not-online-and-why/, dernière consultation le 31 mars 2016.

\section{NOTES}

1. Le mot « surveillance » est associé étymologiquement au verbe "surveiller ", qui détermine un individu dans une position supérieure hiérarchiquement observant minutieusement un autre individu. Cette définition explique la surveillance de type physique. Bien que, dans notre société, la surveillance de type physique existe toujours, dans le cadre des technologies de l'information et de la communication (TIC), la surveillance consiste à «toute méthode de recueil et de traitement de données personnelles, identifiable ou non identifiable, avec pour objectifs d'influencer et de gérer les personnes qui ont fait l'objet de ces procédés " (Lyon, 2001, p. 2, traduction de l'anglais).

2. Par exemple, Kevin Colvin, qui s'est rendu à une fête d'Halloween et a publié une photo de lui déguisé en fée sur Facebook après avoir donné un faux motif d'absence à son employeur. L'un de ses collègues a montré la photo à son supérieur et Kevin a été licencié (Randall et Richards, 2008).

3. Ce sondage est basé sur un échantillon de 860 internautes (Pew Research Center, 2013).

4. Voir www.quitfacebookday.com.

5. Ces chercheurs ont affirmé que des recherches futures au sujet du non-usage des technologies devraient s'intéresser aux logiques d'action liés aux usages, aux non-usages et à la reconnexion à la technologie (Baumer et al., 2013, p. 9). 
6. Différentes méthodes qualitatives ont été utilisées pour mener cette recherche doctorale.

7. Ce formulaire explique aux participants les objectifs, les méthodes, les dimensions éthiques liés au projet, les risques inhérents à leur contribution et les personnes sources à contacter en cas de malaise.

8. «We especially want to avoid imposing categories from the outside that create order and pattern rather than discover it. ».

9. La notion d'amis Facebook est utilisée ici car, du point de vue des usagers, les liens qui les unissent aux individus présents dans leurs cercles sociaux respectifs sur Facebook ne peuvent pas être caractérisés comme des relations d'amitié au sens classique du terme. La majorité d'entre eux sont des connaissances qu'ils ne côtoient plus dans un contexte physique.

10. Un fil où défilent les activités en ligne des amis Facebook et de leurs amis.

11. D’après le dictionnaire Oxford en ligne, le selfie désigne « une photographie de soi prise par soi-même, qui a généralement été prise par un Smartphone ou une webcam et qui a été partagée sur un média social ».

12. L'intégration du profil timeline en septembre 2012 illustre un changement majeur dans son architecture. La timeline présente les activités des utilisateurs, tel un album de photos (scrapbook) depuis leur inscription au site.

13. Proulx et Kwok Choon définissent le contrôle social comme le «processus formel et informel par lequel les normes et conventions qui assurent l'ordre social sont intériorisés par les individus » (2011, p. 106).

\section{RÉSUMÉS}

Cette recherche explore les logiques d'action liées aux usages de Facebook par les jeunes adultes et à la déconnexion temporaire au site. Facebook est utilisé pour pratiquer de la surveillance sociale, mettre à jour son statut et contribuer aux discussions en lien avec les travaux universitaires. Les formes de déconnexion privées sont des conduites de préservation. La reconnexion au site s'effectue car ces usagers succombent aux pressions sociales, par peur de manquer quelque chose (FOMO) et d'être exclus des activités sociales. Les bénéfices liés aux pratiques de socialisation jouent également un rôle en la matière et priment sur le besoin de se protéger de la surveillance. Ces usagers intériorisent doucement le contrôle social. Force est de constater qu'ils ont une connaissance limitée des modes opératoires du site. Il est important de se demander dans quelle mesure la faible visibilité des pratiques de surveillance institutionnelle et les changements constants introduits aux architectures des médias sociaux façonnent les inégalités d'usage. Ce qui contribuerait à la déconnexion, même temporaire.

This research explores motivations linked to the use of Facebook by young adults and temporary disconnection from the site. Facebook is used to practice social surveillance, update status and contribute to discussions related to academic group work. "Private" forms of disconnection aim at protecting the self. Reconnection to the site occurs as users succumb to social pressures, for fear of missing out (FOMO) or being excluded from social activities. The benefits associated with social practices also play a role with regard to reconnection to Facebook and are perceived as more important than protecting oneself from surveillance. These users are internalizing soft social control. However, they have a limited knowledge of the site's operating modes. It is important to know to what extent the limited visibility of institutional surveillance practices and 
the architectural changes introduced to social media sites shape digital inequalities. This may lead to temporary disconnection.

La presente investigación explora las lógicas de actuación vinculadas a los usos de Facebook que realizan los jóvenes adultos y a la desconexión temporal del sitio. Facebook puede ser utilizado para la vigilancia social, para actualizar la situación y para contribuir a las discusiones relacionadas con las tareas universitarias. En este contexto, las formas privadas de desconexión son conductas de preservación. En este sentido, la reconexión al sitio se efectúa porque los usuarios ceden a presiones sociales, o bien por miedo a perderse algo (FOMO en inglés) o por la posibilidad de ser excluidos de actividades sociales. Los beneficios vinculados a las prácticas de socialización también juegan un rol importante en la materia y pesan más que la necesidad de protegerse de la vigilancia, de forma que los usuarios interiorizan el control social. Los usuarios tienen también un conocimiento limitado sobre los modos operatorios del sitio. De esta manera, es importante preguntarse en qué medida la débil visibilidad de prácticas de vigilancia institucional y los cambios constantes introducidos en las arquitecturas de los medios sociales conforman las desigualdades en su utilización. Lo cual también contribuye a la desconexión, incluso temporal.

\section{INDEX}

Mots-clés : Facebook, déconnexion temporaire, logiques d'action, FOMO, contrôle social

Keywords : Facebook, temporary disconnection, motivations, FOMO, social control

\section{AUTEUR}

\section{MARY JANE KWOK CHOON}

Mary Jane Kwok Choon est docteure en communication et chercheuse au Mobile Media Lab à l'Université Concordia. Elle travaille présentement sur le projet ACT (Aging, Communication \& Technologies). Sa thèse a analysé les rapports qu'entretiennent les jeunes à la vie privée quand ils font usage des réseaux socionumériques. Pour plus d'informations sur ses intérêts de recherche, veuillez consulter son blogue : http://maryjanekwokchoon.wordpress.com 